\title{
UNIVERSAL AND NON-UNIVERSAL FIRST-PASSAGE PROPERTIES OF PLANAR MULTIPOLE FLOWS
}

\author{
J. Koplik ${ }^{1}$, S. Redner ${ }^{2}$ and E. J. Hinch ${ }^{3}$ \\ ${ }^{1}$ Benjamin Levich Institute and Department of Physics \\ City College of the City University of New York, New York, NY 10031 \\ ${ }^{2}$ Center for Polymer Studies and Department of Physics \\ Boston University, Boston, MA 02215 \\ ${ }^{3}$ Department of Applied Mathematics and Theoretical Physics \\ Cambridge University, Silver Street, Cambridge CB3 9EW England
}

\begin{abstract}
The dynamics of passive Brownian tracer particles in steady two-dimensional potential flows between sources and sinks is investigated. The first-passage probability, $p(t)$, exhibits power-law decay with a velocity-dependent exponent in radial flow and an order-dependent exponent in multipolar flows. For the latter, there also occur diffusive "echo" shoulders and exponential decays associated with stagnation points in the flow. For spatially extended dipole sinks, the spatial distribution of the collected tracer is independent of the overall magnitude of the flow field.
\end{abstract}

P.A.C.S. Numbers: 47.55.Mh, 05.40.+j

The motion of passive tracer in a flowing fluid is a convenient diagnostic tool for monitoring the characteristics of the flow field, as well as properties of the background medium. In porous media flows, particularly in groundwater or hydrocarbon recovery, tracer measurements often are the only source of internal information about the system [1]. Considerable effort has focused on one-dimensional flows where tracer is introduced at one end of the sample and the transit time distribution is monitored upon exit at the other end. However, in many situations, such as fluid pumped into an "injection" well and extracted from one or more "producing" wells, the flow is more likely to be radial, or multipolar, rather than linear. Our goal, in this letter, is to provide general insights about the first-passage properties for dynamically-neutral tracer in such flows [2].

We shall focus on steady two-dimensional flows which are generated by multipolar configurations of sources and sinks. These appear to encompass many cases of possible physical relevance, as well as illustrating a rich range of phenomenology. Mathematically, the first 
passage of passive Brownian tracer particles is determined by their concentration, $c(\vec{r}, t)$, which satisfies the convection-diffusion equation (CDE),

$$
\frac{\partial c(\vec{r}, t)}{\partial t}+\vec{u}(\vec{r}) \cdot \vec{\nabla} c(\vec{r}, t)=D \nabla^{2} c(\vec{r}, t)
$$

with the velocity field $\vec{u}(\vec{r})$ arising from a two-dimensional potential. We will elucidate the features of $p(t)$, the distribution of transit times or the first-passage probability between source and sink, to characterize the motion of dynamically-neutral tracer in steady flows.

First consider radial or monopolar flow, $\vec{u}(\vec{r})=u_{0} \hat{r} / r$. Non-universal features arise due to the competition between the flow and the centrifugal term in the Laplacian, which both vary as $1 / r$. Consequently, the form of the CDE in two dimensions $(d=2)$ with $u_{0}$ finite is the same as that of a system with no drift but with $d \neq 2$. Thus changing the amplitude $u_{0}$ is equivalent to changing the spatial dimension, leading to non-universal first-passage properties as a function of the drift.

To illustrate these features, we have solved for $p(t)$ in a circular geometry with an inner absorbing radius $a$, infinite outer radius, and an initial ring of tracer particles which are released at $r=r_{0}$. This first passage probability coincides with the radial flux at $r=a$, i.e., $p(t)=-D \partial c(r, t) / \partial r+u(r) c(r, t)$. Using standard Green's function methods, the Laplace transform, $\tilde{p}(s) \equiv \int_{0}^{\infty} p(t) e^{-s t} d t$, is $[2]$

$$
\tilde{p}(s)=\left(\frac{a}{x_{0}}\right)^{\nu} \frac{K_{\nu}\left(x_{0} \sqrt{s / D}\right)}{K_{\nu}(a \sqrt{s / D})} .
$$

where $K_{\nu}$ is the modified Bessel function of the second kind, and $\nu=u_{0} / 2 D \equiv P e / 2$, where $P e$ is the Péclet number. The long-time behavior is determined from the small-s expansion of $\tilde{p}$, which generically has the form, $\tilde{p}(s) \sim \tilde{p}(0)-a s^{\alpha}+b s^{1}+\ldots$ By construction, the leading term $\tilde{p}(s=0)=\int_{0}^{\infty} p(t) d t \equiv E\left(r_{0}\right)$ is just the probability that a tracer particle which starts at $r=r_{0}$ eventually reaches the absorbing circle. Time-dependent properties can be inferred from the correction terms in Eq. (2). When $\alpha<1$, then the mean first-passage time to the absorbing circle, $\langle t\rangle$, diverges and the first-passage probability in real time has a power law tail which varies as $t^{-(\alpha+1)}$. However, when $\alpha$ reaches unity, the coefficient of $-s$ equals $\langle t\rangle$.

For outward flow, the small-s expansion of $p(s)$ is

$$
\tilde{p}(s) \sim\left(\frac{a}{x_{0}}\right)^{2 \nu}\left(1-\frac{\Gamma(1-\nu)}{\Gamma(1+\nu)}\left(r_{0}^{2 \nu}-a^{2 \nu}\right)\left(\frac{s}{4 D}\right)^{\nu}+\ldots\right),
$$

Thus $E\left(r_{0}\right)=\left(a / r_{0}\right)^{2 \nu}<1$ and some tracer escapes to infinity. This reflects the equivalence to a purely diffusive system of spatial dimension $d>2$, for which the eventual return 
probability is less than unity [3]. From the correction term, we deduce that $\langle t\rangle \rightarrow \infty$ for $0 \leq \nu<1$, while for $\nu \geq 1,\langle t\rangle$ is finite. Since $E\left(r_{0}\right)<1$, $\langle t\rangle$ in the case $\nu>0$ is conditional on those particles which actually hit the absorber. Note that for sufficiently large outflow, a tracer particle must reach the absorber in a finite time if it is to be absorbed at all.

For inflow $(\nu<0)$, the first passage probability has the small-s expansion

$$
\tilde{p}(s) \sim 1-\frac{\Gamma(1-\mu)}{\Gamma(1+\mu)}\left(r_{0}^{2 \mu}-a^{2 \mu}\right)\left(\frac{s}{4 D}\right)^{\mu}+\ldots,
$$

where $\mu \equiv-\nu>0$. Thus tracer hits the absorbing circle with certainty, while $p(t)$ is nonuniversal and varies as $t^{-(1+\mu)}$. Additionally for $\mu<1,\langle t\rangle$ is infinite, while for $\mu \geq 1,\langle t\rangle$ is finite. Even though the tracer is guaranteed to reach the absorber for any inflow (including zero), the first-passage time is finite only if the drift is sufficiently strong.

We now discuss tracer motion in a fully two-dimensional dipole flow field due to a point source and sink at $(0, \mp a)$ within a circle of radius $R$, with total flux $\pm Q$. The velocity field may be written as

$$
\vec{u}=\nabla \phi=\left(\frac{\partial \psi}{\partial y},-\frac{\partial \psi}{\partial x}\right)
$$

Here the velocity potential $\phi$ and streamfunction $\psi$ are the real and imaginary parts of the complex potential

$$
\Psi=\phi+i \psi=\frac{Q}{2 \pi} \ln \frac{(z+a)\left(z+R^{2} / a\right)}{(z-a)\left(z-R^{2} / a\right)},
$$

where $\pm R^{2} / a$ are the image locations to impose vanishing normal velocity on the boundary, and $z=x+i y$. We assume that fluid and tracer are emitted uniformly in angle about the source.

In the limit of pure convection in an infinite plane $(R \rightarrow \infty)$, the tracer motion is determined by the velocity field, $d \vec{r} / d t=\vec{u}(\vec{r}(t))$. Since the second equality in Eq. (5) represents a Hamiltonian system, we have applied a canonical transformation technique to compute particle trajectories analytically [4], and thereby determine that the transit time from source to sink for a particle emitted at polar angle $\theta$ with respect to the source is $t(\theta)=2 \csc ^{2} \theta[1-\theta \cot \theta]$; see also [5]. Although the motion is deterministic, a distribution in transit times results from the distribution in initial angle. Since all streamlines lead from source to sink, there is a one-to-one equivalence between the angular and time dependence of the first-passage probability. This leads to

$$
p(t)=p(\theta)\left|\frac{d \theta}{d t}\right| \underset{\theta \rightarrow \pi}{\longrightarrow} \frac{1}{3}(2 \pi)^{-2 / 3} \cdot t^{-4 / 3},
$$

due to tracer particles which initially move away from the sink on distant dipole streamlines. It is this aspect of the distribution which explores the global structure of the flow field. Note that the mean transit time, $\langle t\rangle=\int_{0}^{\infty} d t t p(t)$, diverges. 
To incorporate molecular diffusion, we resort to numerical simulation, and have employed several complementary methods. The simplest is grid-free Monte Carlo time-stepping using individual random walkers, which works best at high Péclet numbers. In time $\Delta t$, a walker is displaced by $\Delta \vec{r}=\vec{u}(\vec{r}) \Delta t+\hat{n}(4 \Delta t / P e)^{1 / 2}$, where $\hat{n}$ is a unit vector of random orientation. Figure 1(a) gives a typical result for $P e=2700$ with $N=500,000$ random walkers. There is an early-time peak, followed by the anticipated $t^{-4 / 3}$ power-law decay, and then a noisy exponential region dominated by diffusion. To clarify the latter domain, we have devised a lattice "probability propagation" algorithm. A probability element at $(i, j)$ translates through a distance $\Delta \vec{r}=\vec{u}(\vec{r}) \Delta t$ in a time $\Delta t$ to an off-lattice position $(x, y)$, and is then redistributed among the 5 -site nearest lattice neighborhood of the target position. The redistribution rule is chosen to ensure that the average displacement of the 5-site group remains equal to $\Delta \vec{r}$ and that diffusion is spatially constant by imposing a constant fluctuation in the displacement of this group. While this method has no statistical fluctuation, the simple form of the redistribution rule restricts the method to low flow rates.

The probability propagation results are equivalent to standard finite-difference methods, but programming is extremely simple. A typical result for $p(t)$ (Fig. 1(b)), exhibits the following four generic features: an early-arrival regime, a power-law decay, a "diffusive echo" shoulder (whose resolution in simulations is dependent on the absence of statistical fluctuations), and an ultimate exponential decay. The shoulder stems from particles which reflect from the boundary before reaching the sink. Because of the low Péclet number involved, non-trivial asymptotic exponent estimation and extrapolation [2] are required to verify the exponent in the power-law region. Since $p(t) \sim 1 /\left(t \ln ^{2} t\right)$ in the diffusive limit [3] and $p(t) \sim t^{-4 / 3}$ as $P e \rightarrow \infty$, it seems unlikely that a simulation method which cannot be applied at large $P e$ will be able to resolve these two limiting behaviors cleanly.

The decay at the longest times may be correlated with stagnation points in the flow field which generically lead to an exponential decay in the transit-time distribution [6]. Without loss of generality, consider a stagnation point at the origin with the local velocity $\vec{u}=d \vec{r} / d t=(-G x, G y)$. If the stagnation point is approached along the $x$-axis, then trajectories which pass near the origin and then escape have the form $\vec{r}(t)=\left(x_{0} e^{-G t}, y_{0} e^{G t}\right)$ with $y_{0} \ll x_{0}$. The time $T$ spent near the stagnation point can be defined by requiring that $\dot{y} \sim U_{0}$, where $U_{0}$ is some characteristic $\mathrm{O}(1)$ velocity. Since $\dot{y} \sim y_{0} G e^{G T}$, this gives $T \sim G^{-1} \ln \left(U_{0} / y_{0} G\right)$. Typically, for trajectories which pass near a stagnation point, the transit time between source and sink is dominated by this value of $T$. The corresponding distribution of transit times may now be obtained by accounting for the distribution of initial positions $y_{0}$. Consequently,

$$
p(T)=p_{0}\left(y_{0}\right)\left|\frac{d y_{0}}{d T}\right|=p_{0}\left(\frac{U}{G} e^{-G T}\right)\left|-U e^{-G T}\right| \underset{T \rightarrow \infty}{\sim} p_{0}(0) e^{-G T} .
$$

Thus the transit time distribution depends on the local shear rate near the stagnation point, 
but not on details of the initial spatial distribution of tracer $p_{0}$. In the present example, applying Eqs. (5), (6), and (8) to the stagnation points at $( \pm R, 0)$ gives an exponential decay rate with $G \propto R^{-3}$. The decay rate observed in simulations will typically be the smaller of this value and the diffusive decay rate, which is proportional to $R^{-2}$ for the dipole case.

For a source-sink of arbitrary multipolarity, the exponent of the power-law tail in $p(t)$ depends on the multipole moment. This exponent can be obtained by the following simple argument. For a $2^{N}$-pole, the leading behavior of the complex potential is $\Psi \sim z^{-N}$, so that the corresponding streamlines are $\psi=\operatorname{Im} \Psi \sim r^{-N} \sin N \theta$, for suitable orientation of the axes. In the convective limit, particle trajectories are defined by $\psi=$ const.. This constraint implies that $\psi(r, \theta)=\psi(R, \pi / 2 N)=R^{-N}$, or $r^{N}=R^{N} \sin N \theta$, where $R$ is the maximum distance from the origin on the streamline. The angular velocity on such a streamline is

$$
\frac{d \theta}{d t}=-\frac{1}{r} \frac{\partial \psi}{\partial r} \sim N r^{N-2} \sin N \theta \sim R^{-N-2} \sin ^{-2 / N}(N \theta) .
$$

The transit time on this trajectory is the time required for $\theta$ to vary between 0 and $\pi / N$. Using the above approximation for the angular velocity, the transit time scales as $t=$ $\int_{0}^{\pi / N} d \theta(d \theta / d t)^{-1} \sim R^{N+2}$. To determine the transit time distribution, we relate the value of $R$ to the initial angle of emission $\theta_{0}$ at the source. Suppose the source is at the origin and oriented so that the long-excursion streamlines are associated with $\theta_{0} \rightarrow 0$. For $r \rightarrow \epsilon$, $\psi=R^{-N} \rightarrow \epsilon^{-N} \sin \theta_{0} \rightarrow \epsilon^{-N} \theta_{0}$, or $\theta_{0} \sim R^{-N}$. Thus the transit time probability distribution is

$$
p(t)=p\left(\theta_{0}\right)\left|\frac{d \theta_{0}}{d t}\right|=\frac{1}{2 \pi}\left|\frac{d \theta_{0}}{d R} \frac{d R}{d t}\right| \sim R^{-2 N-2} \sim t^{-\frac{2 N+2}{N+2}} .
$$

While these predictions have been verified numerically in the convective limit, there are asyet unexplained diffusive features in probability propagation simulations. For example, the form of $p(t)$ for a quadrupole consisting of charges $(-Q, 2 Q,-Q)$ is quite different from that of an "inverted" quadrupole $(Q,-2 Q, Q)$.

Another important diagnostic is the distribution of where on the sink the tracer is collected. In the dipole case, with passive tracer released in steady potential flow between a source and a single spatially extended equipotential sink, we demonstrate that the timeintegrated tracer flux distribution at a given point on the sink is proportional to the incoming fluid velocity at this point. By linearity, this implies that the spatial distribution of the collected tracer is independent of the overall magnitude of the flow. This establishes a useful general equivalence between tracer distributions for pure diffusion and in combined convection and diffusion.

To prove the theorem, we use the complex potential as the conformal mapping to trans- 
form the flow domain from $(x, y)$ into $(\phi, \psi)$. In these variables the CDE becomes

$$
|\vec{u}(\phi, \psi)|^{-2} \frac{\partial c}{\partial t}+\frac{\partial c}{\partial \phi}=\frac{1}{P e}\left(\frac{\partial^{2} c}{\partial \phi^{2}}+\frac{\partial^{2} c}{\partial \psi^{2}}\right) .
$$

This transformation to linear flow introduces a spatially dependent time step which is singular as $(0, i \pi)$ is approached. In the $(\phi, \psi)$ coordinate system, the source has co-ordinate $\phi=\phi_{0}$. For the initial condition of a delta-function pulse of tracer injected at the source, then in the equation of motion for $\xi(\phi, \psi)=\int_{0}^{\infty} d t c(t, \phi, \psi)$, the time derivative term integrates to zero, yielding

$$
P e \frac{\partial \xi}{\partial \phi}=\frac{\partial^{2} \xi}{\partial \phi^{2}}+\frac{\partial^{2} \xi}{\partial \psi^{2}}
$$

with boundary conditions $\left(1-P e^{-1} \partial / \partial \phi\right) \xi=K$, a $P e$-independent constant, at $\phi=\phi_{0}$, and $\xi=0$ at $\phi=\phi_{1}$. The solution of Eq. (12) is $\xi=K\left(1-e^{P e\left(\phi-\phi_{1}\right)}\right)$, and the time-integrated tracer flux arriving at angular position $\psi$ on the sink, $p(\psi)$, is

$$
p(\psi)=-P e^{-1} \int_{0}^{\infty} d t \frac{\partial c}{\partial n}\left(\phi_{1}, \psi, t\right)=-P e^{-1} \frac{\partial \xi}{\partial n}\left(\phi_{1}, \psi\right)=-\left.P e^{-1} \frac{\partial \xi}{\partial \phi} \frac{\partial \phi}{\partial n}\right|_{\left(\phi_{1}, \psi\right)}
$$

where $\hat{n}$ is the unit normal to the sink. In the last expression, the first factor is a constant, and the second is simply the normal velocity of fluid at the sink. Hence $p(\psi) \propto u_{n}(\psi)$ as claimed.

Notice that the theorem also holds in the pure diffusion limit, as can be seen by taking $P e \rightarrow 0$ either in the equation for $\xi$ or in its solution. In this case, the appropriate statement is that $p(\psi)$ is constant for a constant arclength of the sink. Conversely in the limit of no diffusion the theorem is obvious, because then tracer particles remain on their initial streamline, and the tracer flux is simply proportional to the fluid flux. The nontrivial implication is that the local integrated flux to a simply-connected sink is independent of the Péclet number, although the transient flux is Péclet number dependent.

In summary, we have investigated the first-passage probability $p(t)$ of dynamically-neutral tracer in two-dimensional potential flows. For radial flow, convection and diffusion are of the same order leading to non-universal first-passage characteristics. In general multipole flows, the exponent of $p(t)$ depends on the multipole order. The effects of diffusion appear to be subdominant for the dipole but relevant for the quadrupole, as qualitative features of $p(t)$ depend on the sense of the quadrupolar flow when diffusion is present. The influence of stagnation points on the asymptotic properties of $p(t)$ was determined. Finally, the timeintegrated flux to a fixed arclength of sink is independent of the overall magnitude of the flow field. 


\section{Acknowledgements}

We thank Jean Pierre Hulin and Michael Stephen for useful discussions. This research was partially supported by DOE grant DE-FG02-93-ER14327 to JK and NSF grant DMR9219845 to SR.

\section{References}

1. J. Bear, Dynamics of Fluids in Porous Media, (Elsevier, Amsterdam, 1971); F. A. L. Dullien, Porous Media: Structure and Fluid Transport, 2nd ed. (Academic, London, 1991); E. Guyon, J.-P. Nadal and Y. Pomeau, eds., Disorder and Mixing, (Kluwer, Dordrecht, 1988).

2. For full details, see J. Koplik, S. Redner, and E. J. Hinch, "Tracer Dispersion in Planar Multipole Flows", to be published.

3. See, e. g., G. H. Weiss and R. J. Rubin, Adv. Chem. Phys. 52, 363, (1983) and references therein.

4. H. Goldstein, Classical Mechanics, 2nd ed., (Addison-Wesley, Reading MA, 1980).

5. L. Mittag and M. J. Stephen, "Motion and Diffusion of a Passive Scalar in a Two Dimensional Fluid", to be published.

6. P. Kurowski, I. Ippolito, J. P. Hulin, J. Koplik and E. J. Hinch, Phys. Fluids 6, 108 (1994).

\section{Figure Caption}

Figure 1. First-passage probability distribution in combined dipolar flow and molecular diffusion within a circle of radius $R=400$ with source and sink at $x=\mp 20$, obtained by (a) following the motion of individual random walks for $P e=2700$ and (b) probability propagation for Péclet numbers $P e=1.6,0.8,0.4$, and 0.2 (tallest to shortest peak, respectively). The inset in (a) shows the streamlines. Also in (a), the abscissa is the dimensionless time $t \rightarrow t \cdot\left(2 \pi a^{2} / Q\right)$. 